\title{
Campo Geral: Das Fehlerbewusstsein und die Angst der gewaltsamen Wiederholung
}

\section{Erste Überlegungen}

Im Jahr 1956 veröffentlicht Guimarães Rosa das Buch Corpo de baile (dt.: Corps de ballet $)^{1}$, ursprünglich in zwei Bände unterteilt. Im gleichen Jahr veröffentlicht der Autor Grande sertão: veredas (dt.: Grande Sertão) ${ }^{2}$. Corpo de baile besteht aus sieben Geschichten, die von vielen als Novellen, von anderen als Romane bezeichnet werden, wobei diese Gattungszuweisung nicht wesentlich für das Werk ist, wie Rosa in einem Brief an João Condé erklärt, der in der Einleitung des Buches Sagarana veröffentlicht ist: «Wie der weise griechische Lachs von André Maurois sagt: Ein Fluss ohne Ufer ist das Ideal des Fisches.» ${ }^{3}$

Eine der möglichen Arten, Corpo de baile und Grande sertão: veredas als Reihe zu lesen, ist, auf die Wahl des Titels des ersten Buches zu achten. Nach der Theorie des klassischen Tanzes, insbesondere des Balletts, ist unter Corpo de baile (frz. Corps de ballet) das Ensemble der Tänzer der ersten Stufe einer Tanzkompanie zu verstehen, die zusammen mit dem Solisten die Szenen der Vorstellung bilden. So ist die Bühne in den wichtigsten Szenen des Spektakels voll von Tänzern im Hintergrund, sodass der Solist im Zentrum steht. Die gleiche Analogie kann mit den Veröffentlichungen von 1956 gemacht werden. Campo Geral (dt. Miguilim), Uma estória de amor (dt. Eine Liebesgeschichte), A estória de Lélio e Lina (dt. Die Geschichte von Lélio und Lina), $O$ recado do morro (dt. Die Botschaft des Hügels), Lão-Dalalão (dt. Lão-Dalalão), Cara-de-Bronze (dt. Bronzegesicht) und Buriti (dt. Buriti) füllen die Bühne für Grande sertão: veredas, damit dieser als Vertreter für alle hervortreten kann. ${ }^{4}$

1 João Guimarães Rosa: Corps de Ballet. Romanzyklus. Aus dem Portugiesischen von Curt MeyerClason. Köln/Berlin: Kiepenheuer \& Witsch 1966. Die Ausgabe wurde ebenfalls für Zitate aus Buriti verwendet.

2 João Guimarães Rosa: Grande Sertão. Aus dem brasilianischen Portugiesisch von Curt MeyerClason. Köln: Kiepenheuer \& Witsch ${ }^{3} 1994$.

3 João Guimarães Rosa: Sagarana. Rio de Janeiro: Nova Fronteira 2012, S. 23 (unsere Übersetzung, Anm. d. Üb.).

4 Die Titel der deutschsprachigen Übersetzung wurden durch die Übersetzerin hinzugefügt. 
Die erste der sieben Geschichten, die Corpo de Baile ausmachen, trägt den Titel Campo Geral. ${ }^{5}$ In gewisser Weise durchdringen die Erzählelemente dieses Textes alle anderen Geschichten des Buches, wobei es möglich ist, die Charaktere oder einige Hinweise auf den Raum in den anderen Romanen zu finden. Die direkteste Fortsetzung hat man jedoch in Buriti, dem letzten Text, da hier die Hauptfigur Miguilim als Tierarzt Miguel wieder erscheint.

Auch wenn die Hauptfigur ein Kind ist, handelt es sich bei Campo Geral nicht um einen Text, der nur die Freuden des Kindeslebens zeigt. Im Gegenteil, Miguilim muss sich mit extrem komplexen Situationen auseinandersetzen, die seine Identität prägen, welche im Verlauf der Erzählung ausgeprägte Veränderungen erfährt, wodurch neue Perspektiven für den Kleinen aufkommen, so zum Beispiel die konkrete Idee, seinen Herkunftsort Mutúm bei der ersten Gelegenheit, die aufkommt, zu verlassen, und genau das wird er tun:

Seit langem hatte Miguilim keine so große Freude mehr erlebt. Jedoch nicht deshalb, weil er seinen Bruder loswurde. Sondern weil ihm dabei der Gedanke kam: Eines Tages würde auch er von zu Hause fortgehen können. Noch wußte er nicht, wann und wie. Aber der Gedanke hielt ihn hoch wie ein Wort des Trostes. ${ }^{6}$

Aus diesem Grunde zielt die vorliegende Studie darauf ab, einen der Aspekte zu diskutieren, die Miguilims Kindheit am meisten prägen: Gewalt. Sie wird in verschiedenen Formen dargestellt: Demütigungen, körperliche und verbale Aggression, psychologische und symbolische Gewalt. Um die Aspekte von Gewalt als eine Form der Herrschaft des Vaters über seine Familie zu begründen, wird das Werk des französischen Soziologen Pierre Bourdieu mit dem Titel Die männliche Herrschaft verwendet. ${ }^{7}$ Es reflektiert über die Anwendung von vor allem symbolischer Gewalt als Ressource für die Aufrechterhaltung historisch geschaffener Mächte und Hierarchien, die eine Tradition des Missbrauchs und völligen Mangels an gegenseitigem Respekt und Empathie tragen, wobei es sich angesichts dieser von Bourdieu dargelegten Perspektive sowohl um Frauen als auch um Kinder handeln kann, die beide als minderwertig und zerbrechlich angesehen wer-

5 Für die Zitate wurde folgende Ausgabe verwendet João Guimarães Rosa: Miguilim. Übersetzt von Curt Meyer-Clason. Berlin: Klaus Wagenbach ${ }^{3} 2013$. Bei den Angaben werden Seitenzahlen des Originals (wie Anm. 6) und im Anschluss Seitenzahlen dieser Übersetzung genannt. (Anm. d. Üb.)

6 João Guimarães Rosa: Corpo de baile Bd. 1. Rio de Janeiro: Nova Fronteira 2010, S. 143. Üb. S. 129.

7 Pierre Bourdieu: Die männliche Herrschaft. Aus dem Französischen von Jürgen Bolder. Frankfurt a.M.: Suhrkamp 2012. 
den. Da der Schwerpunkt dieser Studie der Charakter Miguilims ist, untersuchen wir eher die Herrschaft des Erwachsenen über das Kind als die des Mannes über die Frau.

\section{Analytische Untersuchung des Romans Campo Geral}

Die Meisterhaftigkeit, mit der Rosa seine Werke verfasst, ist so groß, dass es dem Leser in Texten wie Campo Geral möglich ist, seine Kindheit zu rekonstruieren, indem er sich oft daran erinnert, wie es war, ein Kind zu sein. Diese Rückkehr ist jedoch nicht idealisiert wie es in der Literatur, die sich mit der Welt der Kinder befasst, üblich ist. Oft wird diese Welt perfekt, wunderbar, ohne Probleme dargestellt. Existenzielle Konflikte bestehen lediglich aus der Wahl des nächsten Spiels. Das ist nicht Miguilims Kindheit, und es ist nicht die Kindheit der meisten Leser Rosas, von sehr wenigen Ausnahmen abgesehen. Auf diese Weise erinnern wir uns in Campo Geral daran, wie es war, ein Kind zu sein in einer Welt, in der das Kind keine Stimme hat, abgewertet, verachtet und vor allem nicht verstanden und ständig getadelt wird. Guimarães Rosa erinnert sich an diesen Moment:

Ich spreche nicht gern von der Kindheit. Es ist eine Zeit der guten Dinge, aber immer mit großen Leuten, die uns stören, sich einmischen und uns die Freuden verderben. Wenn ich mich an die Zeit als Kind erinnere, sehe ich zu viele Erwachsene, alle, auch die beliebtesten, wie Soldaten und Polizisten des Eindringlings in einem besetzten Land. ${ }^{8}$

Der Charakter Miguilim stimmt gewissermaßen mit dem Gedanken von Rosa überein. Er sieht die Erwachsenenwelt als nichts sonderlich Schönes: «Miguilim verspürte keine Lust, heranzuwachsen, ein Erwachsener zu werden; die Unterhaltung der großen Leute war immer das gleiche trockene Zeug, sie mußten immer so derb sein, Dinge sagen, die Schrecken einflößten.» ${ }^{9}$ Die Betonung liegt auf dem Wort «mußten〉, als ob die Erwachsenen nicht wirklich das wären, was sie sind. Sie verhalten sich auf diese Weise, weil sie erwachsen sein müssen. Und es ist gerade diese Kindheit voller Konflikte mit den Älteren des Hauses, zu denen die Leser bei der Lektüre des ersten Romans von Corpo de Baile gebracht werden.

8 João Guimarães Rosa: Literatura comentada. São Paulo: Abril Educação 1982, S. 4 (unsere Übersetzung, Anm. d. Üb.).

9 Rosa: Corpo de baile Bd. 1, S. 41. Üb. S. 34. 
Von all den Aspekten, die Miguilim prägen und formen, gewinnen einige an zusätzlicher Relevanz. Unter ihnen lässt sich der Tod des jüngeren und zugleich ihm am nächsten stehenden Bruders Dito hervorheben, der Narben hinterlässt, die noch in Miguels erwachsenem Leben wahrgenommen werden, wie im folgenden Auszug aus Buriti ersichtlich wird: ««Ich habe einen Bruder gehabt, jünger als ich, er ist als Kind gestorben. Ein Brüderchen ...〉 sagte ich. [...] 〈Bis zum heutigen Tag kann ich nie lange an ihn denken, aus Angst, zu leiden. [...]〉.» ${ }^{10}$ Interessanterweise kann erwähnt werden, dass Miguilim andere Brüder hatte: Drelina, Chica, Tomezinho und Liovaldo, aber wenn er gefragt wird, ob er Geschwister hat, erinnert er sich ausschließlich an Dito.

Neben diesem Tod gewinnt ein anderer ebenso negativer Aspekt in Miguels Kindheit an Bedeutung: Gewalt. In diesem Fall ist es die Gewalt der Erwachsenen gegenüber Kindern und in seiner konkretesten Form die des Vaters gegenüber Miguilim. Hervorzuheben ist, dass Gewalt sich nicht nur als körperliche Aggression, sondern auch als psychologische, moralische und symbolische Gewalt versteht. Von daher ist traurigerweise festzustellen, dass der Patriarch der Familie unzählige Ressourcen der Gewalt gegen seine ganze Familie anwendet, wobei Miguilim sein größtes und häufigstes Opfer ist, wie man im folgenden Auszug erkennen kann:

Er stellte sogar fest, daß er niemanden mehr leiden konnte, und nährte einen stillen Zorn auf jeden einzelnen. Besonders auf den Vater. Aber hatte nicht der Vater zuerst einen Haß auf ihn, Miguilim, gehabt? Er brauchte nur Miguilim zu Gesicht zu bekommen, und schon schrie er: «Paß auf, du Lümmel! Topfgucker! Krittler!» In letzter Zeit schmähte er tatsächlich alle. ${ }^{11}$

Der Kleine ist ein sehr sensibles Kind voller Phantasie, das Tiere mag und eine intensive Beziehung voller Zuneigung und Bewunderung zur Mutter hat. Diese Merkmale machen ihn anfälliger für die Gewalttaten des Vaters, insbesondere für die symbolischen, denn in vielen dieser Fälle werden Tiere benutzt, um das Kind zu verletzen. So gewinnt während der Erzählung der Raum mit den Elementen, die ihn darstellen, an Bedeutung und trägt dazu bei, die Perspektive der Hauptfigur darin zu erkennen, so zum Beispiel seine Schwierigkeit, Mutúm als einen schönen Ort zu definieren oder nicht. Diese Charakterisierung ändert sich im Laufe der Geschichte.

Die erste Definition von Mutúm als einem schönen Ort kommt von einem Fremden, den Miguilim auf seiner von Onkel Terêz begleiteten Firmungsreise kennenlernt: Jemand, der in Mutúm gewesen war, hatte gesagt: «Ein hübsches Fleck-

10 Rosa: Corpo de baile Bd. 2, S. 285. Üb. S. 600.

11 Ebda., S. 127. Üb. S. 113. 
chen, zwischen Berg und Berg, mit viel Gestein und Wald, weitab von überall, und es regnet dort immer...» ${ }^{12}$ In diesem ersten Fall wird der Ort, an dem die Familie Cássio lebt, als schön bezeichnet, da der Besucher von der natürlichen Landschaft, den Hügeln, der Vegetation und dem häufigen Regen Notiz nimmt, was darauf hindeutet, dass diese Person während der Überschwemmungszeit, der Regenfälle, in dieser Region war. Es ist bekannt, dass die Jahreszeiten im Landesinneren der Gerais mehrheitlich zweigeteilt sind, die Trockenzeit und die Überflutungszeit, die gerade durch den Gegensatz von Überfluß und Mangel an Regen gekennzeichnet sind.

Diese positive Vision von Mutúm erfüllt Miguilim mit Glück, denn er wusste nur, was die Mutter von dem Ort hielt und die mütterliche Perspektive war schlecht, sie schmerzt die «[...] Traurigkeit, dort wohnen zu müssen. Vor allem klagte sie über die langweiligen Regenmonate, wenn der Himmel sich verdüsterte und alles so einsam, so dunkel wurde; die Luft war dort dunkler, auch in der Zeit der Dürre, an jedem beliebigen Tag, gegen Abend, wenn die Sonne unterging. 〈Oje, oje, was für ein trauriger Winkel〉, stöhnte sie». ${ }^{13}$ Es ist erwähnenswert, dass der Reisende und seine Mutter gleichsam auf die Regenzeit hingewiesen haben, aber für die Frau war sie eine Qual, während sie für den Reisenden genau das war, was Mutúm zu einem schönen Ort machte. Aufmerksamkeit wird also der Subjektivität gewidmet, die die Bewertung des Raumes beinhaltet. Die Vegetation und das Klima sind für alle die gleichen, aber die Beziehung zu allem, was den Ort umgibt, begrenzt die Perspektive jedes einzelnen.

Sobald er kann, erzählt der Sohn der Mutter, was er über die Schönheit von Mutúm gehört hatte, in der Hoffnung, sie würde ihre Sicht ändern, aber «Mutter achtete jedoch nicht darauf, blickte nur wehmütig drein und deutete auf den Berg; sie sagte: «Ich denke immer, daß dort, hinter ihm, Dinge geschehen, die der Berg mir verbirgt, und daß ich sie nie im Leben sehen werde »». ${ }^{14}$ Miguilim ist zu klein, um zu verstehen, warum seine Mutter den Mutúm so negativ sieht:

Nicht etwa weil er, Miguilim, etwas Schönes an Mutúm gefunden hätte, denn er wußte nicht einmal, einen hübschen Ort von einem häßlichen zu unterscheiden. Sondern nur durch die Art, wie der Mann gesprochen hatte, so von weit her, so obenhin, ohne jede Berechnung, und außerdem ganz anders, als seine Mutter es tat, niedergedrückt von Mißmut, unter Seufzern und voller Beschwerde. In der Wurzel von allem steckte ein Irrtum - daß wusste Miguilim, ohne daß er es richtig begriff. Und doch war der Urwald ganz in der Nähe, dunkelgrün, fast schwarz, und flößte ihm Angst ein. ${ }^{15}$

12 Ebda., S. 13. Üb. S. 7.

13 Ebda., S. 13. Üb. S. 7.

14 Ebda., S. 14. Üb. S. 8.

15 Ebda., S. 14-15. Üb. S. 9. 
Aus diesem Auszug wird ersichtlich, dass Miguilim feststellen kann, dass es hinter der Rede der Mutter etwas gibt, das über den einfachen geographisch konstituierten Raum von Mutúm hinausgeht, er wusste "ohne daß er es richtig begriff», dass in der Wurzel von allem ein Irrtum steckt. Ohne genau zu verstehen, erkennt der Junge, dass die Mutter einen weiteren Kontext einschließt, wenn sie den Ort als schön oder hässlich definiert. Innerhalb dieses Kontextes gibt es jedoch einen Fehler. Angesichts dieses Wissens sieht sich Miguilim nicht in der Lage, Mutúm als schön oder hässlich einzustufen. Was für ihn zumindest vorläufig den Ort definiert, ist Angst.

Als sehr sensibler Junge kann Miguilim diese Subjektivität wahrnehmen, auch wenn er sie nicht wirklich begreifen kann. Um besser zu verstehen, fragt der Junge seinen Onkel: «Onkel Terêz, finden Sie, daß Mutúm schön oder häßlich ist?» «Sehr schön, Miguilim, was denn sonst! Ich wohne gern hier.» ${ }^{16}$ Wieder ist der Junge mit einer subjektiven Antwort konfrontiert, der Onkel antwortet nicht «schön» mit Bezug auf die Vegetation, das Klima und die Tiere der Region, was die Schönheit oder Hässlichkeit eindeutig definieren würde. Es ist schön, dort zu leben, was zusätzlich durch den in Minas Gerais populären Ausdruck «uai» untermalt wird, was der Antwort einen Beiklang von Offensichtlichkeit verleiht: «Muito bonito, Miguilim; uai. Eu gosto de morar aqui...».

So durchdringt Miguilim die gesamte Erzählung mit Versuchen, Mutúm als einen hässlichen oder schönen Ort zu definieren. Bei diesen Versuchen kann man erkennen, dass sich die Perspektive des Kindes in Bezug auf den Ort verändert, wenn sich die Elemente, die ihn darstellen, verändern: die Menschen, das, was er gerade fühlt und was das alles darstellen könnte.

Das erste Mal, als er den Ort definiert, stuft er ihn als hässlich ein: «Mutúm war ein trauriger Flecken, es war öde und häßlich. Der Hügel, dunkler Buschwald mit all seinen bösen Tieren, lag auf der Lauer, von dort her drang auch das Gekrächz der Geier.» ${ }^{17}$ Was den Jungen zu dieser Beurteilung bringt, ist wieder die Angst. Der Junge weiß noch nicht, dass er Sehprobleme hat, aber die fehlende Bestimmung dessen, was wahrgenommen wird, macht ihm Angst, deshalb fürchtet er sich derart vor dem Wald. Die großen und unförmigen Bilder, die ihn im Unbekannten erwarten, würden Angst in jedem Kind auslösen. Aus diesem Grund wird dieser Ort als hässlich empfunden. Ein Ort, der ein so unbequemes Gefühl repräsentiert wie die Angst, könnte wohl nicht anders eingestuft werden als traurig und hässlich.

16 Ebda., S. 16. Üb. S. 10.

17 Ebda., S. 67. Üb. S. 58. 
Die Angst kommt hauptsächlich mit der Dunkelheit, die die Formen, vor allem für Miguilim, noch vager werden lässt. Tagsüber war die Art, Mutúm zu sehen, jedoch anders. In erster Linie aufgrund eines ganz bestimmten Aspekts: der Abwesenheit des Vaters. Es ist auffällig, wie sehr die Abwesenheit des Vaters die Kinder beruhigt, besonders Miguilim. Weil er ruhiger ist, fühlt er sich auch glücklicher. Die Wahrnehmung spiegelt sich in der Außenwelt als «innere Landschaft» wider. Der Ort erhält neue Farben und neue Bewertungen: «Jene Tage waren schön, es fiel kein Tropfen Regen, es gab nur Sonnenschein, und es grünte sommerlich. Vater brachte die ganze Zeit auf dem Feld zu und schuftete wie ein Sklave - so sagte jedenfalls Mama. Es war gut für die Kinder, daß der Vater nicht zu Hause war.» ${ }^{18}$

Die Tatsache, dass der Vater nicht anwesend ist, ist dermaßen auffallend, dass selbst die Nacht, von ihren erschreckenden Mysterien bedeckt, sich von ihrer schöneren Seite zeigen kann: «So wurde jener Abend, ohne Papa und Großmama Izidra, zum schönsten aller Tage. Es war Vollmond, und abends sagte Mama, sie wollten alle einen Spaziergang machen, wohin und wie lang man wollte.» ${ }^{19}$ Der schönste Tag von allen ist für den Jungen nicht zufällig der Tag, an dem Vater und Großmutter väterlicherseits, die zwei höchsten Symbole des Konservatismus und des Patriarchats, nicht zu Hause sind. Die Familie, die die Position des Traditionellen einnimmt, ist oft die Grundlage für die Denkweise der Herrschaft des Mannes über die Frau und des Erwachsenen über das Kind. Wie Bourdieu betont: «Die Hauptrolle bei der Reproduktion der männlichen Herrschaft und Sicht fällt sicherlich der Familie zu.» ${ }^{20}$ Der Vater führt in diesem Fall die Herrschaft an sich und in gewalttätiger Form aus, und Großmutter Izidra übernimmt bereits die Rolle der Verewigung dieser Herrschaft, nicht durch direktes gewalttätiges Eingreifen, sondern durch die Bewahrung dieser Werte und ihrer Nachgiebigkeit. In diesem Fall wird der Raum schöner, gerade weil diese beiden Figuren fehlen und mit ihnen die Symbolik des dominanten und gewalttätigen Patriarchats.

Erst am Ende der Erzählung kann Miguilim definieren, was er von Mutúm hält:

Und Miguilim blickte sie alle an, mit aller Kraft. Und lief hinaus. Und blickte hinauf zu den dunklen Wäldern auf der Hügelkuppe, hier das Haus, den Zaun aus Wildbohnen und São Caetano; den Himmel, den Korral, den Quintal; die runden Augen und die hohen Glasfenster des Morgens. Er blickte weiter hinaus, sah das Vieh weiden am Moor, bedeckt mit Wasserlili-

18 Ebda., S. 99. Üb. S. 88-89.

19 Ebda., S. 102. Üb. S. 91.

20 Bourdieu: Die männliche Herrschaft, S. 148. 
en wie mit Watte. Sah das Grün der Buritis am ersten Flußlauf. Ach, Mutúm war schön, nun wußte er es. ${ }^{21}$

Miguilim findet Mutúm jetzt schön, oder besser gesagt, er weiß jetzt, dass Mutúm schön ist. Damit er das feststellen kann, werden mehrere Faktoren berücksichtigt, von denen der wichtigste die Fähigkeit ist, richtig sehen zu können. Miguel trägt eine Brille und zum ersten Mal gibt es keine gruseligen Schatten im Busch, er kann sehen, dass es sich nur um Bäume und einige Tiere handelt. Außerdem gibt es die Figur des Vaters und all die Gewalt, die ihn umgibt, nicht mehr, denn der Vater ist tot. Darüber hinaus verlässt Miguilim Mutúm und zieht in die Stadt. Dabei handelt es sich um den Moment, in dem sich das Leben des Jungen abspaltet. Er wird keine Kopie des Vaters sein. Er hat die ganze Zukunft vor sich angesichts des Unbekannten, jedoch in dem positiven Glauben, dass alle Gewalt mit dem Vater begraben worden ist.

Damit bricht der Junge mit einem sich wiederholenden, zur Unendlichkeit neigenden Zyklus. So wie sein Vater wohl auf der Grundlage von Gewalt erzogen wurde und so zu einem gewalttätigen und unterdrückenden Mann geworden ist, ist Miguilim zum Zeitpunkt der Erzählung acht Jahre alt. Aus der Erzählung heraus werden Tendenzen ersichtlich, dass er allmählich so aggressiv werden würde wie der Vater. Nicht selten beginnt der Junge, nachdem er einige Prügel erlitten hat, Hassgefühle zu schüren, es kommt sogar soweit, dass er daran denkt, den Vater zu töten. Wahrscheinlich würde er auf diese Weise groß werden und aufhören, der sensible Junge zu sein, der er war und zur Fortsetzung der väterlichen Figur werden, die Gewalt als eine Form der Herstellung von Beziehungen nutzt, um diese männliche Herrschaft aufrechtzuerhalten. Über diese Verewigung der Unterdrückung sagt Bourdieu:

\footnotetext{
Es ist in der Tat klar, daß das Ewig-Währende in der Geschichte nichts anderes sein kann als das Ergebnis einer geschichtlichen Verewigungsarbeit. Das bedeutet, daß es, um dem Essentialismus zu entgehen, nicht darum zu tun sein kann, das Dauerhafte und Invariante zu leugnen, das unzweifelhaft einen Teil der geschichtlichen Wirklichkeit ausmacht. Vielmehr gilt es, die Geschichte der geschichtlichen Enthistorisierungsarbeit zu rekonstruieren oder, wenn man das vorzieht, die Geschichte der fortdauernden (Wieder-)Herstellung der objektiven und subjektiven Strukturen der männlichen Herrschaft, die sich, seit es Männer und Frauen gibt, permanent vollzieht und durch die die männliche Herrschaft kontinuierlich von Generation zu Generation reproduziert wird. ${ }^{22}$
}

21 Rosa: Corpo de baile Bd. 1, S. 155. Üb. S. 140.

22 Bourdieu: Die männliche Herrschaft, S. 144. 
So haben wir die historische Beschaffenheit von Unterdrückung und Gewalt «seit es Männer und Frauen gibt>. Diese Situation wird zur Ewigkeit, solange sich soziale Strukturen nicht verändern. In diese Strukturen passt die Familie als eines der Zahnräder, die den unterdrückenden Patriarchalismus bewahren. Wenn also die Cássio-Familie sich nicht verändert hätte, wäre nur die Fortsetzung der Gewalt zu erwarten, aber das ist nicht der Fall. Die Geschichte wird aus dem Selbstmord des Vaters und dem Umzug von Miguilim in die Stadt neu konstruiert. Was der erwachsene Miguel am meisten gefürchtet hatte, war die Wiederholung, wie im Auszug aus Buriti zu lesen ist: «Wenn Miguel Angst verspürte, war seine Lebensangst die Angst vor der Wiederholung.» 23 Die Angst, dass alles so werden würde wie es bereits war, Angst, dass sich die Geschichte wiederholen würde. Aufgrund dieser zwei nachdrücklichen Veränderungen eröffnet sich die Möglichkeit der Nichtwiederholung, des Bruchs mit dem Ewigen.

Miguilim ist zunächst kein gewalttätiger Junge, im Gegenteil, er nimmt Anteil an tierischem Leid, das oft Ziel der Scherze der Erwachsenen ist, die angesichts des Tierleids dieselbe Kälte zeigen, wie gegenüber des Leids von Kindern, das sie nicht dulden. Das erste Beispiel dafür, dass Miguilim anders ist als alle Anderen, findet sich in Campo Geral in den Erinnerungen an den Umzug der Familie nach Mutúm:

\footnotetext{
Einer der Brüder, er wußte nicht mehr welcher, trank Ziegenmilch; daher wurde die weiße Ziege an einer Stange hinter dem Wagen hergezogen. Die Zicklein reisten mit den Menschen im Wageninnern und blökten unablässig nach ihrer Mutter. Die arme Ziege - musste sie nicht bald umfallen vor Müdigkeit? «Fein, die hat die Euter zum Bersten voll» sagte jemand. Warum ließen dann die Allerärmsten die ganze Milch unterwegs über Stock und Stein und Staub fließen ${ }^{24}$
}

Der Erzähler hebt die Idee hervor, dass die Szenen, die Miguilim in Erinnerung blieben, irgendwie mit der Natur in Verbindung stehen, wie in dem oben genannten Zitat, in dem das Leiden der Ziege dargestellt wird, die angebunden an den Ochsenkarren mitläuft, ohne das geringste Recht auf eine Pause und darüber hinaus mit vollem Euter laufen muss, dessen Milch sich über die Steine auf dem Weg ergießt. Diese Erinnerungsszene beschreibt nicht nur die Reise nach Mutúm. Sie ist geprägt von der Bosheit, mit der das Tier behandelt wird, und dem mangelnden Mitgefühl der Erwachsenen.

23 Rosa: Corpo de baile Bd. 2, S. 319 .Üb. S. 629.

24 Rosa: Corpo de baile Bd. 1, S. 18. Üb. S. 12. 
Diese sehr subtil erscheinende Form der Gewalt ist in der gesamten Erzählung sehr üblich. Der Vater übt seine Herrschaft über die Familie durch physische und symbolische Gewalt aus, wobei es sich hier um den ersten möglichen Fall handelt, den man in der Beziehung zwischen Vater und Miguilim erkennen kann. Symbolische Gewalt bedeutet zunächst aus der Perspektive Bourdieus:

Es ist jene sanfte, für ihre Opfer unmerkliche, unsichtbare Gewalt, die im wesentlichen über die rein symbolischen Wege der Kommunikation und des Erkennens, oder genauer des Verkennens, des Anerkennens oder, äußerstenfalls, des Gefühls ausgeübt wird. Diese soziale Beziehung, die so außerordentlich gewöhnlich ist, bietet daher eine besonders günstige Gelegenheit, die Logik einer Herrschaft zu erfassen, die im Namen eines symbolischen Prinzips ausgeübt wird, das der Herrschende wie der Beherrschte kennen und anerkennen. Dabei kann es sich um eine Sprache (oder Aussprache), einen Lebensstil (oder eine Denk-, Sprechoder Handlungsweise) und, allgemeiner, eine distinktive Eigenschaft, ein Emblem oder ein Stigma handeln, unter denen die symbolisch wirksamste die Hautfarbe ist, diese völlig willkürliche körperliche Eigenschaft ohne jede Voraussagekraft [...]. ${ }^{25}$

So besteht die Beziehung zwischen Vater und Sohn in der Frustration und dem Unverständnis des Kindes vor der Haltung, die der Vater einnimmt, wie zum Beispiel, wenn er einigen Passanten, die dort vorbeikommen, den Hund Pingo-deOuro mitgibt. Der Vater hatte wahrscheinlich die Zuneigung des Kindes für das Tier bemerkt und die Hündin weggegeben, wobei es sich um ein Merkmal von symbolischer Gewalt und Beherrschung handelt, die der Vater auf das Kind ausübt:

In Miguilims Augen stand jedoch Pingo-de-Ouro an der Spitze, eine Seele von einer Hündin, die hier keinen Herrn hatte, ihn aber vor allen anderen bevorzugte. Wenn er sich hinten im Gemüsegarten versteckte, um allein zu spielen, kam sie einhergetrottet, ohne ihn zu belästigen, ohne zu bellen, blieb still in der Nähe und schien zu verstehen. Sie war immer knochenmager, von kränklicher Natur, es hieß, sie würde bald erblinden. Aber sie bekam Junge. [...] Bald darauf kamen etliche Treiber durch Mutúm und blieben einige Tage, weil fast alle ihre Maultiere lahmten. Als sie weiterzogen, gab ihnen Miguilims Vater die Hündin mit auf den Weg, die sie an einem Strick mitzerrten; der Welpe jaulte in einem Korb hinter ihr her. Sie ritten, wohin es sie rief. Miguilim weinte herzzerreißend, seine Trauer kannte keine Grenzen, immer wieder rannten die Tränen. Jemand sagte, es sei schon vorgekommen, daß ein verschenkter Hund, viele Léguas weit entführt, wieder heimgefunden habe. Nun faßte er Hoffnung: Pingo-de-Ouro würde wiederkehren. Geduldig wartete und wartete er. Sogar nachts, wenn irgendwo ein Hund bellte, glaubte er, sie sei es. Wer würde ihr aufmachen? Sie mußte müde sein, durstig, hungrig. «Die findet nicht mehr heim, die war ja schon fast blind.» Wenn sie also fast blind war, warum hatte sie dann der Vater fremden Leuten mitgegeben ${ }^{26}$

25 Bourdieu: Die männliche Herrschaft, S. 8.

26 Rosa: Corpo de baile Bd. 1, S. 21. Üb. S. 14-15. 
Die Zweifel am Ende, «Wenn sie also fast blind war, warum hatte sie dann der Vater fremden Leuten mitgegeben?», bringen die Inkohärenz der väterlichen Haltung an die Oberfläche, die sich als grundlos herausstellt und fast ausschließlich der Aufrechterhaltung der Herrschaft dient. Sie besteht leiglich darin, dem Jungen zu zeigen, wer der Stärkere in der Situation ist. Im herausgehobenen Abschnitt ist es möglich, auf subtile und indirekte Weise die Anwesenheit einiger Dialoge zwischen Miguilim und einigen Erwachsenen wahrzunehmen, unter denen möglicherweise die Rede des Vaters ist. Aufgrund der Traurigkeit des Jungen - «Miguilim weinte herzzerreißend, seine Trauer kannte keine Grenzen, immer wieder rannten die Tränen» - weckten sie Hoffnung in ihm «Jemand sagte, es sei schon vorgekommen, daß ein verschenkter Hund, viele Léguas weit entführt, wieder heimgefunden habe.» Dieser 〈Jemand〉 könnte einer der Männer gewesen sein, die draußen auf den Ackern mit seinem Vater gearbeitet haben und Mitleid mit dem Kind bekamen. Aber eine andere Stimme, wahrscheinlich die des Vaters, macht es sich zur Aufgabe, den Traum des Kleinen, Pingo-de Ouro könnte eines Tages zurückkehren, zu zerstören: «Die findet nicht mehr heim, die war ja schon fast blind...» Die große Frage, die sich Miguilim stellt, ist: Warum? Wenn sie wussten, dass sie fast blind ist, warum haben sie sie weggegeben? In dieser erzählten Welt werden die Fragen der Kinder jedoch nicht von den Erwachsenen beantwortet, und angesichts Miguilims Leiden herrscht nur Schweigen.

Mit einem solchem Verhalten stärkt der Vater seine Position als herrschsüchtiges und unbestrittenes Familienoberhaupt und lässt keinen Platz für offene Gespräche, Aufmerksamkeit oder Zuneigung. Die Wünsche der Kinder werden nicht nur nicht respektiert, sie werden nicht einmal gehört und somit bewegen sich die Institutionen mittels symbolischer Gewalt auf die Fortdauer von Gewalt und Patriarchat hin, wobei die männliche, väterliche Figur eine herausstechende Stellung in dieser Konstruktion einnimmt. Zusätzlich zu all dieser symbolischen Gewalt benutzt der Vater andere Tricks, um Furcht in seinen Sohn zu erwecken, wie die Drohungen, ihn im Wald anzubinden:

\footnotetext{
Aber der Vater hätte nicht sagen sollen, daß er ihn, Miguilim, einmal ganz schlimm bestrafen und am Waldrand an einen Baum binden würde. Wenn sie das täten, würde er da nicht aus Angst ersticken? Aus dem Wald oberhalb des Hügels kam doch der Jaguar. Wie konnte der Vater sich nur eine so schlimme Strafe ausdenken und ein Kind im pechschwarzen Wald an einen Baum festbinden wollen? Nur der Vater von Hänsel und Gretel, in dem Märchen Vater und Mutter führten die beiden tief in den Wald hinein, damit sie sich nicht mehr auskannten, denn die Eltern hatten nichts mehr zu essen für sie. Miguilim empfand so großes Erbarmen mit Hänsel und Gretel, daß ihm wieder die Tränen kamen. ${ }^{27}$
}

27 Ebda., S. 25. Üb. S. 19. 
Es ist wichtig zu betonen, dass der Vater diese Strafe eben genau deshalb vorschlug, weil er Miguilims Angst vor dem Wald kannte. Diese Tatsache erhöht die Raffinesse der Grausamkeit des Patriarchen erheblich. Die Infragestellung des Erzählers wiederholt sich, dessen Stimme mit Miguilims verschmilzt: Welche Bosheiten konnte der Vater sich nur noch vorstellen, wenn er einen Jungen in der Dunkelheit im Wald anbinden wollte? Die Situation verursacht Befremdlichkeit im unschuldigen Denken eines Kindes. Ein Erwachsener jedoch, von dem eigentlich anzunehmen wäre, dass er der rationalere Part der Beziehung ist, sollte sich viel mehr über die Unangemessenheit dieser Bestrafung im Klaren sein, die dazu beiträgt, dass der Kleine sich sehr vor der Situation fürchtet.

Gerade deshalb, weil er sich der Präsenz dieser Angst bewusst ist, droht der Vater Miguilim mit dieser Strafe. Er benutzt psychologische Gewalt und Terror angesichts des Vollzugs dessen, was ihn am meisten mit Entsetzen erfüllt, um ihn zu beherrschen. Diese ausgewachsene Grausamkeit erzeugt allmählich Hass in Miguilim. Zuerst in Bezug auf den Vater, dann aber in Bezug auf alle Anwesenden einschließlich der Mutter, die er zwar verehrt, aber auch sie sieht Miguilim bald mit anderen Augen, da sie keine Position zum Schutz ihrer Kinder bezieht: «Aber Miguilim mochte auch seine Mutter nicht mehr leiden. Mutter litt zwar seinetwegen, aber sie war weich und verteidigte ihn nicht, sie wollte nicht seinetwegen, des kleinen schwachen Jungen, streiten.» ${ }^{28}$

Die Formen der Gewalt, die der Vater gegen Miguilim anwendet, variieren und durchlaufen Demütigungen, Demoralisierungen, verbale und physische Aggressionen bis hin zur symbolischen Zerstörung von Miguilims Verbundenheit mit dem, was ihm am besten gefiel: den Tieren, besonders den Vögeln.

Diese Aggressivität des Vaters ist völlig zwecklos und unangemessen. Es gibt kein Gleichgewicht zwischen Fehlverhalten und Bestrafung. Jegliches Verhalten, wie unbedeutend es auch sein mag oder wie zufällig auch immer es sich entwickelt, ist eine hinreichende Rechtfertigung dafür, dass Miguilim streng bestraft wird, was bei dem Jungen das Gefühl der Ungerechtigkeit verursacht und dadurch auch Gefühle von Wut und Hass in ihm weckt.

Die erste große Erniedrigung ergibt sich an einem Tag, an dem die Jungen auf Bäume klettern. Beim Abstieg reißt die Hose von Miguilim:

[...] nur seine Hose zerriß, und zwar der Länge nach. Alles war noch gut abgelaufen. Als der Vater kam, polterte er los, man solle dem Jungen die Hose flicken. Und befahl ausdrücklich, Miguilim solle zur Strafe nackt stehenbleiben, ohne Hose, bis die Mutter den Riß zugenäht habe. Schon das genügte, daß man vor Scham halb umkam. Hatte man denn kein Mitleid mit ihm, mußte man so hart mit ihm ins Gericht gehen [...]? Ah, wenn es keine Sünde gewe-

28 Ebda., S. 136. Üb. S. 122. 
sen wäre, er hätte einen Mordszorn auf Papa bekommen, auf alle zusammen, ein Zorn voller Haß, denn er hatte ja allen Grund dazu. ${ }^{29}$

In keinem Moment will Miguilim die Hose vorsätzlich zerreißen. Das passiert wie bei jedem Kind, wenn es spielt. Der Vater hätte den Jungen schelten können, vorsichtiger oder aufmerksamer zu sein, wenn er auf Bäume klettert oder herumspielt. Doch die Einstellung des Vaters grenzt an Irrationalität. Den Jungen vor allen nackt stehen zu lassen, bis Mutter die Kleider näht, ist eine völlig unnötige und unverhältnismäßige Bestrafung in Bezug auf den Vorfall. Miguilim ist sich bewusst, dass der Vater die Grenzen überschritten hat, so sehr, dass der Erzähler bestätigt, «er hatte ja allen Grund dazu». Die ersten Funken des Zorns gegenüber der Vaterfigur kommen im Jungen auf. Aber dieses Gefühl wird bald von dem Jungen selbst mit der Vorstellung der Sünde gezügelt. Für eine religiöse Familie, und Miguilim ist sehr gläubig, wie Oma Izidra in einem bestimmten Moment in der Erzählung hervorhebt, ist die Vorstellung der Sünde wichtig und veranlasst ihn, seine Wut auf den Vater zu beschwichtigen. Doch die religiöse Weltanschauung reicht nicht aus, um zu verhindern, dass der Junge extreme Gefühle für den Erwachsenen hegt.

Es ist durchaus üblich, dass in religiösen Kontexten einige Missbräuche, insbesondere in Bezug auf hierarchische Strukturen im Namen des eigenen Glaubens vertuscht werden, wie Bourdieu betont:

[...] hat sie [die Kirche] sich auf ein System von ethischen Gegensätzen stützen können, das einem bestimmten kosmologischen Modell entsprach, um die Hierarchie innerhalb der Familie als eine auf die Autorität des Vaters gegründete Monarchie von göttlichem Recht zu rechtfertigen und [...] eine Sicht der sozialen Welt [...] durchzusetzen. ${ }^{30}$

So kann die Vorstellung der Sünde für eine Zeit Miguilims schlechtes Gefühl für den Vater zügeln, aber die Demütigungen und Aggressionen hören nicht auf, was das Kind dazu bringt, sich von der totalen Hingabe an die Hierarchie, die als göttlich angesehen wird, zu lösen und den Hass, den es für seinen Peiniger empfindet, zu nähren.

Der Vater erniedrigt ihn nicht nur körperlich, sondern auch moralisch. Vor jedem, auch dem Jungen selbst, bedauert der Vater den Tod Ditos, dass eigentlich Miguilim hätte sterben sollen: «Aber abends, zu Hause, sagte er zu Mutter in Miguilims Gegenwart, er tauge nicht, Dito hingegen sei ein braves Bürschchen gewe-

29 Ebda., S. 58-59. Üb. S. 51.

30 Bourdieu: Die männliche Herrschaft, S. 129. 
sen, aber Gott habe ihn zu sich genommen, er hätte lieber Miguilim holen sollen statt Dito.» ${ }^{31}$ Der Junge beginnt auf dem Hof zu arbeiten und tut alles, um die Bestätigung des Vaters zu bekommen, aber durch seine Sehschwäche scheint er für erwachsene Augen zerstreut zu sein, weil der Junge oft in Löcher fällt und über Stöcke und Steine stolpert. Aber der Patriarch ist nicht fähig, sich in die Situation des achtjährigen Jungen hineinzuversetzen, der aufs Feld gehen und mit den Älteren der Routine nachgehen muss. Anstatt zu versuchen, einige Situationen, die das Kind betreffen, nachzuvollziehen oder zumindest aufzuklären, demoralisiert der Vater das Kind und trägt nach und nach dazu bei, dass die Beziehung zwischen ihnen immer distanzierter und mit zunehmend schwerer zu lösenden Problemen behaftet wird.

Der Höhepunkt körperlicher Aggression kommt zu einer Zeit, in der Miguilim selbst schon aggressiver wird. Als er sieht, wie sein Bruder Liovaldo den kleinen Grivo schikaniert, geht Miguilim auf den älteren Bruder los und schlägt auf ihn ein, er überrascht alle mit seiner Kraft. In der Folge verpasst ihm der Vater mit der Absicht, ihn wegen der Gewalt zu schelten, die größte Tracht Prügel, die Miguilim je bekommen hatte. Dieses völlig widersprüchliche Verhalten - schließlich ist es ungewöhnlich, einem Kind mittels einer Tracht Prügel beizubringen, dass er niemanden schlagen darf - ist der Wendepunkt für Miguilims Beziehung zu seinem Vater und zu Mutúm selbst:

Er packte Miguilim und schleppte ihn unter Püffen nach Hause, unter den Vorbau. Dort versetzte er ihm ein paar Ohrfeigen, dann zog er ihn pudelnackt aus und begann ihn mit seinem Leibriemen zu traktieren. Er prügelte und schimpfte, was das Zeug hielt, und gefiel sich dabei. Er hämmerte so wild auf seinen Sohn ein, daß Mama, Drelina und Chica, Rosa, Tomèzinho, sogar Großmutter Izidra weinten, flehten, er solle endlich aufhören, nun reiche es. Aber der Vater ließ nicht locker, ließ die Riemenschläge auf Miguilim niedersausen, aber Miguilim vergoß keine Träne. Er weinte nicht, weil er sich an einen Gedanke klammerte: Sobald er erwachsen war, würde er Vater totschlagen. Er dachte darüber nach, wie er Vater umbringen würde, und nun begann er zu lachen. Und schon ließ Vater los, verdutzt, verblüfft; da er aber auch auf Miguilims Kopf getrommelt hatte, dachte er, Miguilim sei vielleicht verrückt geworden. ${ }^{32}$

Die Aggressivität des Vaters ist enorm und betont die Reaktion des Mannes, wenn er das Kind schlägt. «Er prügelte und schimpfte, was das Zeug hielt, und gefiel sich dabei.» Alle im Haus, einschließlich Oma Izidra, die immer dazu beigetragen hatte, die Hierarchie und männliche Herrschaft im Hause zu bewahren, weinten und flehten den Vater an, mit dem exzessiven Schlagen aufzuhören. Dann ent-

31 Rosa: Corpo de baile Bd. 1, S. 130. Üb. S. 117.

32 Ebda., S. 136. Üb. S. 122. 
scheidet sich Miguilim: Wenn er erwachsen ist, wird er den Vater töten. Die Wut des Jungen ist so groß, dass er lacht, als er sich vorstellt, wie er das machen könnte. Das positive Band der Beziehung zwischen ihnen ist zerrissen und Hass nimmt den Platz ein, den eigentlich Zuwendung und Respekt einnehmen sollten. Was Miguilim wieder zum Lächeln bringt, ist die Idee, Mutúm so schnell wie möglich zu verlassen, um so vor den Unterlassungen der Mutter und der Grausamkeit des Vaters zu flüchten.

Die Grausamkeit der Erwachsenen ist jedoch kein exklusiver Charakterzug des Vaters. Andere Erwachsene zeigen den gleichen Mangel an Verständnis für kindliche Ängste, wie Miguilim es ausdrückt. Wie bereits oben erwähnt, müssen sie brutal sein. Dieser Aspekt wird im Moment der Jagd auf die Gürteltiere deutlich, eine Aktivität, die in der Fazenda Routine ist und Miguilim sehr stört:

[...] Was sie aber am meisten jagten, waren Gürteltiere; draußen wimmelte es von ihnen. [...] Sie waren fett und gerissen, und trotzdem mußten anscheinend alle sterben, wollten die Leute ihnen allen denn den Garaus machen? [...] Miguilim brannte darauf, daß das Gürteltier heil davonkommen möchte. ${ }^{33}$

Die Sorge des Erzählers fällt mit Miguilims Gefühl der Hoffnung zusammen, dass das Gürteltier unversehrt der Jagd entkommen wird, weil er nicht versteht, weshalb so viele Gürteltiere getötet werden müssen:

In der Nähe, gleich oberhalb des Korrals, hatten sie einmal ein behaartes Gürteltier gefangen - wie das grunzte! -, das Gürteltier mit dem flachen Kopf kreischt am lautesten, wenn es sich von den Hunden gestellt sieht. Die Hunde trieben es in die Enge, umstellten es, es war ein Weibchen und rollte sich auf, winselte, versuchte ein Loch zu Graben. Die Hunde ließen es nicht zu. Sie wälzten sich mit ihm am Boden, rasch kam es wieder auf die Füße. Man sah, daß es rennen konnte wie ein Wiesel, hätten die Hunde es gelassen. Weiße Härchen wuchsen zwischen seinen Panzerschuppen wie die letzten feinsten Wurzelspitzen. Und es hob die Pfoten, gekreuzt, zeigte seine Fingernägel, die wie verkrustete Knochen aussahen. Es bat um Mitleid. Später, bei einer anderen Gelegenheit, war es kein Sechsbindengürteltier, sondern ein Neunbindengürteltier, das noch schneller läuft und ein richtiger Renner ist. Es schnaubt, wenn es von einem Hund gepackt wird. Vater zog sein Messer, legte nach und stach zu. Es zischte Izúis, Izúis ...! Es war schon am Verenden und scharrte noch immer mit den Krallen im Erdboden, als wolle es sich eingraben. «Die verdienen kein Mitleid, Miguilim, die verdammten Viecher fressen einem den ganzen Mais vom Feld weg, sie stoßen die Stengel um, nagen die Kolben an, sie graben das Saatgut aus, bloß um zu fressen», sagte Viehtreiber Salúz, um ihn zu trösten, «die Tiere treiben es gar zu arg, das Gürteltier frisst auch Wurzeln.» Warum machten sich dann Papa und die anderen immer so ein Gaudium daraus, warum lachten, lärmten und tobten sie immer, sobald es darum ging, irgend etwas zu jagen, das Gürteltier und irgendwelche anderen wehrlosen Tiere zu töten? Mit diesem Heißhunger, mit

33 Ebda., S. 29. Üb. S. 22. 
dieser rotglühenden Lust ergötzte sich wohl der Teufel in der Hölle an den Leiden der Menschen! Sie wollten nicht, daß Miguilim Erbarmen mit dem Gürteltier fühlte, mit dem armseligen Gottesgeschöpf, das allein auf weiter Flur war und keinen Freund hatte. Miguilim lernte noch eine neue Art des Widerwillens gegen die großen Leute. Er mochte noch so erwachsen werden, nie würde er sie lieben lernen, nie ihr aufrichtiger Freund sein. Und wenn er einmal groß war und die anderen eines Tages sich mauserten, um gut zu sein, sie hatten trotzdem einmal ein Gürteltier zu Tode gequält, hatten Freude und hämische Lust dabei empfunden, sie hatten die Cuca Pingo-de-Ouro fortgeschickt, weiß der Himmel wohin, wo sie niemanden erkennen würde, zumal sie fast blind war. ${ }^{34}$

Die Beklemmung, mit der der Erzähler die Situationen beschreibt, ist dieselbe, mit der Miguilim all dies erlebt. Der Fokus liegt auf dem Kind. Die Leser leiden angesichts solcher Grausamkeiten mit ihm. Der Junge und der Erzähler humanisieren die Tiere - in diesem Fall das Gürteltier - gänzlich. Menschen und Tiere stehen beinahe auf gleicher Höhe, mit dem Unterschied, dass der Junge gegenüber den Erwachsenen Widerwillen empfindet: «Miguilim lernte noch eine neue Art des Widerwillens gegen die großen Leute. Er mochte noch so erwachsen werden, nie würde er sie lieben lernen, nie ihr aufrichtiger Freund sein», während er für die Gürteltiere Mitleid empfindet, was in den beschriebenen Bewegungen des in die Enge getriebenen Gürteltiers deutlich wird: «- es war ein Weibchen und rollte sich auf, winselte», «Und es hob die Pfoten, gekreuzt, zeigte seine Fingernägel, die wie verkrustete Knochen aussahen. Es bat um Mitleid...» «Es zischte Izúis, Izúis...!» Ein Gürteltier, oder besser ein Weibchen, das winselt, die Arme hebt und Mitleid erregt, und noch ein weiterer Gefährte derselben Art, den er Jesus nennt, diese sind des Mitgefühls würdig. Es sind Tiere mit menschlichen Merkmalen und sind de facto menschlicher als all die anderen Erwachsenen dort, schon weil die Tiere die Maispflanzen «bloß um zu fressen...» platttreten, was für die Männer ein nicht zu rechtfertigendes Argument ist, aber welches dann jegliche Handlung rechtfertigt. Das stellt Miguilim in Frage. Erwachsene jagen und töten das Gürteltier nur zum Spaß, worin Miguilim den Sadismus erkennt, der die ganze Situation beinhaltet: «Warum machten sich dann Papa und die anderen immer so ein Gaudium daraus, warum lachten, lärmten und tobten sie immer, sobald es darum ging, irgend etwas zu jagen, das Gürteltier und irgendwelche anderen wehrlosen Tiere zu töten?» Er vergleicht den Sadismus mit der Jagd als eine vom Teufel gemachte: «Mit diesem Heißhunger, mit dieser rotglühenden Lust ergötzte sich wohl der Teufel in der Hölle an den Leiden der Menschen!»

Die Erwachsenen wissen, dass Miguilim Mitleid mit den Tieren hat und weisen dies zurück: «Sie wollten nicht, daß Miguilim Erbarmen mit dem Gürteltier

34 Ebda., S. 63-64. Üb. S. 55-56. 
fühlte.» Sie bringen den Jungen in peinliche Situationen, mit der Absicht, ihn zu peinigen und zu provozieren, wie in folgender Situation deutlich wird:

\begin{abstract}
Dann kamen die Viehtreiber von ihrer Arbeit zurück und erzählten, die Hunde hätten ein Riesengürteltier gestellt, ein Riesenvieh! «Es schleuderte Steine und Erde hinter sich, und zwar solche Mengen, daß ihm niemand nahe kommen kann. Und selbst wenn jemand drauftritt, hört es nicht auf zu graben.» «Das Vieh hat eine unheimliche Kraft!» stimmte Viehtreiber Jé zu. Sagte, es gebe Leute, die Gürteltiere nicht essen mögen, weil ihr Fleisch nach Blumen schmeckt. «Aber das Fleisch der anderen Gürteltiere schmeckt mit Farofa sehr gut!» Miguilim mußte über so viel Geschwätz lachen. Ohne nachzudenken, fragte Viehtreiber Jé: «Wenn ich es mit Farofa koch, Miguilim, magst du’s dann essen? Hast du dann kein Mitleid mit dem Gürteltier mehr?» «Natürlich habe ich Mitleid mit ihm, und zwar viel. Ich habe nur nicht dran gedacht.» Und Miguilim wurde wütend, weil man ihm die Frage gestellt hatte. ${ }^{35}$
\end{abstract}

Es ist bemerkenswert, dass Miguilim wütend über die Provokation ist. Er lacht über die «Poesiererei», die Art der Viehtreiber, zu sprechen, die in ungewöhnlicher Manier das Fleisch des Gürteltiers mit dem Geschmack einer Blume vergleichen. Aber der Mangel an Sensibilität und die menschliche Boshaftigkeit werden durch die Erwachsenen herausgefiltert, die es nicht für notwendig halten, sich in die Rolle der Kinder zu versetzen und mit dem scherzen, was sie für eine Schwäche des Jungen halten: das Mitleid für die Tiere.

Miguilim ist ein Junge, der extrem mit den Tieren verbunden ist, also leidet er mit ihnen und kann an ihnen auch Seiten wahrnehmen, die über das hinausgehen, was die Sicht des Erwachsenen gewöhnt ist. So sehr, dass manchmal die Phantasie mit dem geschichtenerzählenden Jungen durchgeht und er ein menschliches Leben auf die Tiere projiziert, z. B. als Onkel Terêz mit einem auf der Jagd erlegten Kaninchen nach Hause kommt und der kleine Miguilim an das Leben denkt, das der Hase führte und wie ungerecht sein Tod gewesen ist:

Jeden Tag wurden auf der Jagd geschossene Tiere heimgebracht. Der Feldhase hatte seine
Höhle am Waldrand, verließ sein Versteck aber nur beim Dunkelwerden, dann wollte er fres-
sen, wollte spielen, humpelpumpel, ein Häschen aus dem Wald, es schürzte sein Schnäuz-
chen auf allerhand Art, hüpfte ulkig umher und hockte sich auf seinen Hintern, aufrecht,
aufmerksam, die Löffel bibbernd und bebend. Er mußte doch einen Spielkameraden haben,
Männchen oder Frauchen, oder ein Brüderchen das nun am Waldrand auf ihn wartete, ganz
allein. ${ }^{36}$

Die Unschuld des Jungen im Umgang mit den Tieren wird in gewisser Weise zu seiner Schwachstelle. Denn dies ist der hervorstechende Punkt, der dem Vater als

35 Ebda., S. 100. Üb. S. 89.

36 Ebda., S. 28. Üb. 21. 
eine Möglichkeit dient, das Kind zu bestrafen. Nachdem der Patriarch der Familie sieht, dass seine Prügel nicht mehr wirksam sind, appelliert er an eine andere Art von Gewalt und zerstört ohne Scham oder Groll das, was für das Kind am wichtigsten ist: seine Verbindung zu Tieren. Er erkundet grausam diese Eigenschaft von Miguilim, wodurch er den Jungen auch zu dessen Höhepunkt von Gewalt und Hass bringt.

\begin{abstract}
Aber Vater schlug nicht zu. Er tat etwas anderes, er nahm die Käfige, einen nach dem anderen, ging hinaus, öffnete sie und ließ die Vögel in die Freiheit fliegen, Miguilims Vögel. Dann zertrampelte und zerstückelte er die Vogelkäfige. Alle waren stumm wie das Grab. Miguilim rührte sich nicht von der Stelle. Vater hatte sämtliche Vögel davon fliegen lassen, auch das Kronfinkenpärchen, das Miguilim einmal aus eigenem Antrieb und Einfall mit einem Sieb an der Küchentür gefangen hatte. Miguilim wartete, ob Vater doch noch auf ihn losgehen würde. Aber er tat es nicht. Dann ging Miguilim hinaus und lief ans Ende des Gemüsegartens, wo er ein Wasserrad gebaut hatte, trat darauf und zerstörte es. Er lief zum Cajueiro, an dem die Vogelfallen hingen, riß sie ab und zerstörte sie. Dann ging er und sammelte alle seine Spielsachen ein, alles, was er aufbewahrt hatte [...] und warf alles in den Hof. Daraufhin ging er in den Kornspeicher, um dort noch mehr Wut zu bekommen. ${ }^{37}$
\end{abstract}

Mit diesem Verhalten kann der Vater Miguilim in seinem tiefsten Inneren treffen. Mit dem Wissen, dass Prügel nichts mehr nutzen, dass Erniedrigung nicht mehr genügt, appelliert der Vater an die emotionale Seite des Sohnes und bricht ihn symbolisch, indem er jeden Käfig zerbricht und jeden Vogel freilässt. Miguilims Reaktion besteht nicht darin, auf den Vater loszugehen, sondern die Handlungen des Patriarchen zu wiederholen, indem er zerstört, was ihm geholfen hat, seine Kindheit aufzubauen. Er zerbricht die Spielsachen und wirft all die Dinge weg, die aus der Kindheit noch überlebt hatten, die aber aufgegeben worden waren, «um [...] noch mehr Wut zu bekommen.»

Zweifellos ist dies das Verhalten des Vaters, das Miguilim am meisten verändert, ihn dazu bringt, das Kindsein aufzugeben und ihn in das aggressive Leben der Älteren überführt. Der Junge hört auf, sich wie ein Achtjähriger zu verhalten, der Geschichten erzählt, spielt und sich eine andere Zukunft vorstellt. Miguilim denkt jetzt nur noch an den Tag, an dem er jenen Ort verlassen kann. Er arbeitet hart auf den Feldern, damit der Vater keinen Grund hat, ihn zurechtzuweisen, er nährt den Hass auf den Vater, bis zu dem Punkt, an dem der Tod des Vaters ihn kaum mehr berührt. Und die Furcht, die ihn dazu treibt, die Aktivitäten aus Angst vor Missbilligung sehr sorgfältig zu machen, verfolgt ihn durch sein Erwachsenenleben hindurch, wie in dem folgenden Ausschnitt aus Buriti zu sehen ist: «Miguel hatte stets eine geheime Angst, zu trödeln oder etwas falsch zu ma-

37 Ebda., S. 141-142. Üb. S. 127-128. 
chen. Einen Horror, daß er Fehler machte, daß Fehler vorkamen. Als könnte ihm plötzlich jemand in der Hitze des Arbeitstages oder in der Kühle des Abends einen heftigen Vorwurf, eine ungerechte Zurechtweisung an den Kopf werfen.» ${ }^{38}$

Es ist also festzustellen, dass der Tod von Miguilims Vaters von grundlegender Bedeutung ist, sodass die Wiederholung, vor der sich der Junge so gefürchtet hatte, nicht eintrifft. Hinzu kommt, dass aufgrund der Möglichkeit, zum Studieren in die Stadt zu gehen, beim erwachsenen Miguel einige Merkmale wiedergeboren werden, die zunichte gemacht worden waren, wie sein Mitgefühl und seine Aufmerksamkeit für die Tiere sowie die Fähigkeit, auf gesunde Weise mit anderen Menschen Beziehungen einzugehen, ohne die Notwendigkeit, Gewalt oder Grausamkeit anzuwenden. Aufgrund dieser Faktoren wird Miguel schließlich Tierarzt und beschließt, Glorinha, ein schönes Mädchen aus dem Hinterland, zu heiraten.

\section{Abschließende Überlegungen}

Campo Geral ist ein Roman, der den Leser sehr bewegt, indem dieser Miguilim und seine Geschwister ein Stück in deren Kindheit begleitet. Die Verbundenheit der Jungen und die Neugierde derer, die die Zukunft noch vor sich haben, trifft auf die Gewalt der Institution Familie, die vom Vater repräsentiert wird, einer Figur maximaler Autorität, die ihren Kindern kein Band der Zuneigung und Liebe weitergeben kann. Diese Verbindung fordert er mittels Gewalt.

Der Vater arbeitet hart, damit die Kinder etwas zu essen haben, er leidet an der Krankheit der Kinder, aber er weiß nicht mit Autorität umzugehen und vermischt sie mit Autoritarismus. So wendet er unverhältnismäßig gewaltsame Strafen für die von den Kindern begangenen Delikte an. Diese Aspekte konstituieren die Komplexität des Charakters und auch die komplexe Kindheit von Miguilim, die gerade durch die exzessive Repression und die Gewalt der Erwachsenen gekennzeichnet ist.

Miguilim hat Angst vor Wiederholung, Angst vor der Verewigung des Fehlers, aber im Laufe der Erzählung verwandelt das Verhalten des Vaters gegenüber dem Jungen diesen in den aggressiven Spiegel der männlichen Figur des Hauses. Am Ende jedoch wendet sich diese Gewalt gegen den Patriarch selbst, der sein eigenes Leben angreift, indem er sich selbst aufhängt, nachdem er seinen Kollegen getötet hat. Mit diesem Tod löst sich die traditionelle Familieninstitution auf. Es ist ein Wandel, der durch die Abreise von Großmutter Izidra, die zusammen mit

38 Rosa: Corpo de baile Bd. 2, S. 298-299. Üb. S. 161. 
dem Vater die hierarchische männliche Ordnung der Unterdrückung im Haus aufrecht erhalten hatte, und Miguilims Weggang in die Stadt intensiviert wird.

Diese drei Faktoren erlauben, dass sich die Geschichte von Miguilim nicht wiederholt, der Fehler nicht bewahrt wird. Er wird Tierarzt und kann einige seiner Eigenschaften wieder entfalten, die ihn in der Kindheit ausmachten, die aber von den oppressiven Haltungen des Vaters mit seinen Demütigungen, physischen, psychologischen und symbolischen Aggressionen unterdrückt worden waren. Guimarães Rosa verfasst damit einen hochreflexiven Text, der sich nicht auf eine simple oder idealisierte Weise der Kinderwelt nähert, sondern aus der Perspektive des Kindes zeigt, wie es ist, dort zu sein, keine Stimme zu haben und von Erwachsenen unterdrückt zu werden. Am Ende eröffnet sich sogar das hoffnungsvolle Tor der Veränderung, die mit der Genealogie männlicher Herrschaft bricht. 